Revised Manuscript BONE-D-09-00585

\title{
Temporal Pattern of Gene Expression and Histology of Stress Fracture Healing
}

Kidd $L J^{\text {ad }}$, Stephens $A S^{b}$, Kuliwaba JS ${ }^{c}$, Fazzalari $N L^{c}, W u A C K^{a}$ and Forwood MR

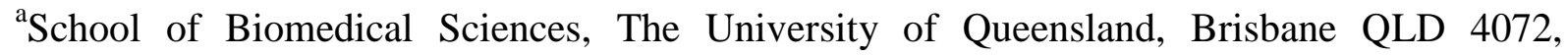
Australia

bschool of Medical Science, Griffith University, Gold Coast QLD 4222, Australia

${ }^{\mathrm{c} B o n e}$ and Joint Research Laboratory, SA Pathology and Hanson Institute, Adelaide SA 5000,

Australia

${ }^{\mathrm{d}}$ School of Veterinary Science, The University of Queensland, Brisbane Qld 4072

Running head: Healing of stress fractures

Number of Figures: 4

Number of Tables: 4

Number of words/characters: Abstract 269/1399; Manuscript 6800/36,900

Email addresses: 1.kidd@uq.edu.au; a.stephens@griffith.edu.au;

julia.kuliwaba@imvs.sa.gov.au; nick.fazzalari@imvs.sa.gov.au; a.wu@uq.edu.au

Address for Correspondence

Prof Mark R Forwood PhD

School of Medical Science

Griffith University

Gold Coast Qld 4222

Australia

Tel +61 755527305

Fax +61 755528908

Emailm.forwood@griffith.edu.au 
Funding: This work was funded in part by NHMRC Project Grants 301143, 401553 and 453624; a University of Queensland Graduate School Scholarship (LK), and The Rebecca L. Cooper Medical Research Foundation 


\section{Abstract}

Loading of the rat ulna is an ideal model to examine stress fracture healing. The aim of this study was to undertake a detailed examination of the histology, histomorphometry and gene expression of the healing and remodelling process initiated by fatigue-loading of the rat ulna. Ulnae were harvested 1, 2, 4, 6, 8 and 10 weeks following creation of a stress fracture. Fracture healing involved direct remodelling that progressed along the fracture line as well as woven bone proliferation at the site of the fracture. Histomorphometry demonstrated rapid progression of basic multicellular units from 1-4 weeks with significant slowing down of healing by 10 weeks after loading. Quantitative PCR was performed at 4 hours, 24 hours, 4 days, 7 days and 14 days after loading. Gene expression was compared to an unloaded control group. At 4 hours after fracture there was a marked, 220-fold increase $(\mathrm{P}<0.0001)$ in expression of IL-6. There were also prominent peak increases in mRNA expression for OPG, COX-2, and VEGF (all $\mathrm{P}<0.0001$ ). At 24 hours there was a peak increase in mRNA expression for IL-11 (73-fold increase, $\mathrm{P}<0.0001$ ). At 4 days there was a significant increase in mRNA expression for Bcl-2, COX-1, IGF-1, OPN, and SDF-1. At 7 days there was significantly increased mRNA expression of RANKL and OPN. Prominent, up-regulation of COX-2, VEGF, OPG, SDF-1, BMP-2 and SOST prior to peak expression of RANK-L indicates the importance of these factors in mediating directed remodelling of the fracture line. Dramatic, early up-regulation of IL-6 and IL-11 demonstrate their central role in initiating signalling events for remodelling and stress fracture healing.

Key Words: stress fracture; bone remodelling; microdamage; gene expression; cytokines 


\section{Introduction}

Stress or fatigue fractures are incomplete, non-displaced fractures that occur following repetitive loading, rather than a single traumatic event $[1,2]$. They are common injuries in human and animal athletes and affect children, adolescents and adults at all levels of training [3-6]. Stress fractures can occur in osteoporotic bone when loading within normal physiologic ranges is imposed on bone with inadequate mechanical properties [1, 7]. Although there is now considerable information available on the epidemiology and incidence of stress fractures, there is almost no objective information on the repair processes involved in the healing of clinical stress fractures. As many fractures occur in otherwise healthy patients, and most fractures are managed conservatively, biopsy material is very rarely obtained [8]. Fracture healing has been classically described as proceeding via direct repair or via callus formation and subsequent endochondral ossification.

Highly stable and compressed fractures can heal by gap healing or direct remodelling across the fracture line without callus formation [9, 10]. As stress fractures are incomplete, it is likely that they heal by primary bone healing with remodelling across the fracture line but the lack of rigid compression and some continued use of the affected limb in most patients may determine the pattern of healing involved. Stress fracture management involves a combination of exercise modification, immobilisation and/or surgical intervention. Many fractures are slow to resolve, some can progress to complete fractures and refractory healing, similar to nonunions, have been reported [11-14]. A better understanding of the repair processes involved is vital to enable more rational approaches to stress fracture management and improved outcomes following treatment. 
Repetitive loading of the rat ulna has become a popular approach to investigate bone fatigue injury and remodelling [15-18]. This model has been used to create experimental fatigue fractures and these fractures are associated with woven bone formation and loss of mechanical properties [19, 20]. Undecalcified histology has demonstrated resorption spaces associated with fractures following fatigue loading [15, 21]. We have optimised the use of this model to develop a repeatable, non-invasive and clinically relevant model of stress fracture in the rat that provides an invaluable opportunity to investigate stress fracture healing. Our aim was to investigate the cellular processes occurring during stress fracture healing by using histology and histomorphometry to quantify bone formation, resorption and remodelling over a 10week period after loading.

Gene expression data have been reported in rat models of complete fracture and intramembranous bone formation [22, 23]. Tissue and vascular damage, release of growth factors from the extracellular matrix, upregulation of many cytokines and migration of inflammatory and endothelial cells are involved in the healing of complete fractures [9, 24]. Wohl et al (2009) examined gene expression for a number of factors associated with bone formation and angiogenesis during woven bone formation associated with stress fracture in the rat ulna. However, the cellular signalling events associated with the remodelling response to stress fracture healing have not previously been examined. We hypothesise that the mechanism of healing will involve different molecular pathways to that of complete fracture healing. Therefore, quantitative gene expression studies will provide unique information on the cascade of signalling events occurring during stress fracture healing. In addition, if fatigue fractures created in the rat ulna are repaired by directed remodelling, rather than endochondral ossification, this model will provide a valuable approach to examine the temporal relationship between the initiation of bone resorption and expression of mediators of bone remodelling. There is convincing evidence that remodelling "targets" microdamage caused by fatigue 
loading of bone $[1,15,25]$. Osteocyte apoptosis, direct osteocyte damage or interruption of canalicular connections between cells by microcracks have been reported as possible stimuli for local remodeling responses to fatigue loading and microdamage [26-30]. This remodelling process is critical to bone's ability to adapt to its environment. Inappropriate remodelling and bone resorption are central to a number of important clinical conditions including osteoporosis, stress fractures and osteoarthritis, which are characterised by impaired structure $[1,7,31]$.

We aimed to measure the temporal pattern of expression of a panel of genes known to affect bone cell function to determine their importance and timing during directed, or targeted, healing of an in vivo model of fatigue fracture. If stress fractures are repaired by direct remodelling, defined as remodelling that occurs independently of endochondral ossification and callus formation, then the signalling molecules required to originate the basic multicellular units (BMUs) should be consistent with those classically associated with chemotaxis and osteoclastogenesis.

We hypothesise that stress fractures produced in the rat ulna will heal by direct remodelling along the stress fracture line, and that initiation of a stress fracture elicits a cytokine cascade that involves upregulation of IL-6, IL-11 and RANKL in a hierarchical manner, and downregulation of osteoprotegerin, resulting in the initiation of direct bone resorption of the stress fracture.

\section{Materials and methods}

\section{Experimental animals}

A total of 98 Female Wistar rats weighing 270 to 360 grams and aged 5 months were used (University of Queensland Biological Resources Unit). Rats were housed in pairs and allowed ad libitum feeding and unrestricted cage activity before and after loading sessions. All 
experiments were carried out under guidelines and approval from The University of Queensland animal ethics committee. A single injection of an opioid analgesic (Buprenorphine $0.05 \mathrm{mg} / \mathrm{Kg}$ subcutaneously) was used following loading sessions. No evidence of lameness or behavioural changes was seen following loading. Euthanasia was performed using $\mathrm{CO}_{2}$ asphyxiation.

\section{Loading model}

Loading sessions were conducted by placing the flexed carpus and elbow in cups and applying axial cyclic loading on the ulnae of rats using methods similar to those described elsewhere [15, 20]. Halothane and Oxygen general anaesthesia was used. Loading was performed in a custom designed loading device which has previously been described in detail [32]. To determine displacement in the limb during loading, the loading device was attached to a linear vertical displacement transducer (LVDT) connected to a MacLab (AD Instruments, Colorado Springs, USA) and monitored using Chart v5.4 (AD Instruments, Colorado Springs, USA). An oscilloscope connected to the load controller was used to confirm the load magnitude and loading as a Haversine wave. Loading was applied as cyclic compressive loading at 17 to $24 \mathrm{~N}$ load and $2 \mathrm{~Hz}$ cycle frequency. The load range was selected based on previous reports in the literature using similar sized rats, and commenced at $17 \mathrm{~N}$. In all rats the displacement value decreased over the first 1000 to 2000 cycles, and then stabilised, until a rapid increase in displacement leading up to fatigue failure. Loading was manually stopped at the point when a $10 \%$ increase in displacement was reached, compared to the lowest measured displacement rather than the initial displacement. The total number of cycles to fatigue ranged from 4000 to 12000 cycles. 


\section{Histomorphometry and Histology}

Because complete fractures in rats take 6-8 weeks to heal [9] we examined histology samples from 1 to 10 weeks after loading to investigate the timing and nature of stress fracture healing. We used decalcified histology to provide greater detail of cellular activity within the BMU along the healing stress fracture, and to allow precise serial location of the region for histomorphometric analysis. Undecalcified histology enables measurement of flourochrome labels and bone formation indices, but does not allow for differentiation of resorption and formation activity within the BMU during healing along the fracture line. Decalcified sections allowed detailed analysis of the resorption activity within the BMUs, and as well as indirect assessment of bone formation during healing. The right ulna was loaded in 50 rats. Rats were euthanised at 2, 4, 6, or 10 weeks after loading $(n=15)$. Subsequently, groups were included at 1 and 8 weeks $(n=5)$ to verify the initiation of resorption, woven bone formation, and continuity of remodelling.

A $10-15 \mathrm{~mm}$ section of the right ulna containing the fracture was removed from the bone and fixed in cold $10 \%$ neutral buffered formalin for 2 days and stored in $70 \%$ ethanol until processed. Bones were decalcified for 7 weeks in 14\% EDTA (pH 7.2) before being embedded in paraffin using standard protocols. Multiple $5 \mu \mathrm{m}$ thick transverse sections were obtained along the full length of the fracture line. Toluidine Blue and Saffranin-O staining were performed. Examination of multiple serial sections along the entire length of the fracture was undertaken for qualitative assessment of fracture healing.

TRAP staining was performed on 3 sections per bone to confirm the presence of osteoclasts in sections. The staining solution ( $\mathrm{pH} 5.0$ ) consisted of $0.1 \mathrm{M}$ Tris- $\mathrm{HCl}, 17.5 \mathrm{mg} / \mathrm{mL}$ napthol AS-BI phosphoric acid (Sigma), $128 \mathrm{mg} / \mathrm{mL}$ hexasotized pararosaniline (Sigma) reacted with $128 \mathrm{mg} / \mathrm{mL}$ of sodium nitrite and $1 \mathrm{mg} / \mathrm{mL}$ of sodium tartrate. The sections were incubated with the stain in a $37^{\circ} \mathrm{C}$ oven for 60 minutes and were counterstained with Mayer's 
Hematoxylin and mounted in Cytoseal permanent mounting media. TRAP positive cells were not counted because the sections used for special staining were not obtained from a standard location within the ulnar diaphysis, but simply used for qualitative verification of cell morphology.

Histomorphometry was performed on one Toluidine Blue-stained section from each bone at a standard level along the fracture. This section was the point where the fracture line was half way between the medial cortical margin and the medullary cavity of the bone in transverse section. Histomorphometry measurements were obtained using a digitising pad (Wacom Intuo 2, Vancouver, WA) and KSS software (KSS Stereology, Salt Lake City, UT). The area of a BMU was defined as the total area that had been resorbed. Within this BMU, the area filled with new bone formation was defined as healed area. This healed area was identified by a distinct cement line around a previously resorbed area of bone. The area that had been resorbed, but not yet filled by new bone formation, was defined as porosity. Measurements obtained were cortical area (Ct.Ar, $\mathrm{mm}^{2}$ ), woven bone area (Wo.B.Ar, $\mathrm{mm}^{2}$ ), length of fracture (SFx.Le, $\mu \mathrm{m}$ ), total length of BMU along the fracture line (BMU.Le, $\mu \mathrm{m}$ ), percentage of fracture length occupied by BMU (BMU.Le/SFx.Le.\%), length of healed fracture line (SFx.He.Le $\mu \mathrm{m}$ ), length of fracture line occupied by porosity (SSFx.Po.Le $\mu \mathrm{m}$ ) percentage of fracture line healed (SFx.He.Le/SFx.Le.\%), percentage of fracture line occupied by porosity (SFx.Po.Le/SFx.Le.\%), total area of BMUs associated with the fracture line (BMU.Ar, $\mu \mathrm{m}^{2}$ ), total perimeter of the BMUs (BMU.Pm, $\mu \mathrm{m}$ ), area of porosity within the BMUs (SFx.Po.Ar, $\mu \mathrm{m}^{2}$ ), area of healed new bone within the BMUs (SFx.He.Ar, $\mu \mathrm{m}^{2}$ ) and area of cartilage within the woven bone (Ca.Ar, $\mu \mathrm{m}^{2}$ ). 


\section{Gene expression}

To investigate the temporal pattern of signalling events related to the early initiation of bone remodelling, we examined gene expression over the first 2 weeks after loading. Quantitative real-time PCR (qPCR) was performed on the left and right ulnae from 40 rats in which a stress fracture was created in the right ulna. Rats were euthanised 4 hours, 24 hours, 4 days, 7 days and 14 days after loading ( $n=8$ /group). A control group ( $n=8)$ did not undergo loading and real time-PCR was performed on only the right ulna in this unloaded control ULC group. The proximal and distal physis and articulation were removed from the left and right ulnae and the remaining diaphysis of each bone was dissected free of all soft tissue, except for periosteum, and snap frozen in liquid nitrogen. Ulnae were stored at -80 degrees for 1-4 weeks prior to processing.

For total RNA extraction, individual ulnae were ground to a fine powder in liquid nitrogen in a mortar and pestle. The bone powder was added to $1 \mathrm{ml}$ of Trizol ${ }^{\circledR}$ (Invitrogen), homogenised using a pipette tip and then centrifuged at $12000 \mathrm{~g}$ for 10 minutes to remove insoluble and mineralised material. Total RNA was then isolated using the manufacturer's instructions and the RNA pellet was resuspended in $8 \mu$ l of DEPC-water. The total RNA yield from the ulna diaphysis was approximately $0.5 \mu \mathrm{g}$. For each sample, the entire quantity of RNA was treated with DNAseI (Invitrogen) to remove any residual DNA and then converted to cDNA using Superscript II (Invitrogen) with oligo (dT) primers. The samples were not pooled and real-time-PCR's were performed for each bone in duplicate with a total volume of 20 4 l using pre-made TaqMan assays (Applied Biosystems, Foster City, CA)(Table 1) in an ABI PRISM 7500 Detection system. Gene expression data were expressed relative to GAPDH internal control using the relationship $2^{-\Delta C T}$. 
The quantity of mRNA isolated from each bone allowed for RT-PCR analysis of 20 different genes. Genes were selected to examine signalling associated with regulation of bone resorption (RANK, RANKL, OPG, TNF $\alpha$, IL-1 $\alpha$, IL-6, IL-11), bone formation (BMP-2, Runx-2, IGF-1, SOST), cartilage formation and endochondral ossification (Collagen type 2 and 10, noted as collagen-2 and collagen-10, respectively), apoptosis (BAX, Bcl-2), angiogenesis (VEGF) chemotaxis (SDF-1), or with multiple potential functions in bone biology (COX-1, COX-2, OPN).

\section{Statistical analysis}

Histomorphometry

All variables had a normal distribution using a Kolmogorov-Smirnov test. Differences between timepoints for each variable were determined using a one-way ANOVA and post hoc Fisher’s protected least significant difference (LSD) analysis.

Gene expression

Fold changes between the right (loaded) ulnae group and the opposite left ulnae internal control group were determined for each timepoint and paired t-tests were performed to determine the significance of differences in the means if the change between loaded and unloaded was greater than twofold. As there was the potential for systemic effects to cause increases in gene expression in the contralateral, unloaded, ulnae, data from either the left or right ulnae timepoint groups were compared to the unloaded control group. All variables were checked for normal distribution using a Kolmogorov-Smirnov test. Normally distributed variables in the right limb groups were VEGF, BAX, OPG, OPN, TNF $\alpha$, RANK, RANKL and COX-2. Normally distributed variables in the left limb groups were BAX, Bcl-2, RANK, SOST, SDF-1, IGF-1, VEGF and Runx-2. These variables were analysed using separate oneway ANOVAs. The differences between left or right timepoint groups and the control group 
were determined using post hoc Fisher's protected (LSD) to compare means between groups. Variables that were not normally distributed for the right limb groups were Bcl-2, COX-1, IL1, IL-11, BMP-2, Collagen-2, Collagen-10, IGF-1, IL-6, Runx-2, SDF-1 and SOST. For the left limb groups variables that were not normally distributed were COX-1, COX-2, IL-1, IL11, BMP-2, collagen-2, collagen-10, IL-6, TNF $\alpha$, OPG, OPN and RANKL. These variables were analysed using the non-parametric Mann-Whitney test to compare left or right timepoint groups against the unloaded control group. For all tests, significance was determined at $\mathrm{P}<$ 0.05 .

\section{Results}

All rats showed normal behaviour and moved freely with minimal signs of lameness after loading. They all ate and drank normally following loading with no change in food or water intake.

\section{Fatigue fracture configuration}

All 98 loaded rat ulnae developed stress fractures. Fracture configuration and woven bone response were ascertained by direct visual examination of the bone and by histological examination of serial sections. Fatigue fractures occurred in the distal half of the diaphysis and extended 3-7 mm within the cortical bone. All fractures were a "U" shape on the medial (compression) surface of the bone. On the transverse sections most fractures originated as a semi-circular "exit point" on the cortex becoming wider and straighter as they extended obliquely towards the medullary cavity.

\section{Histology}

Woven bone (WoB) was seen on all the bones that incurred a stress fracture (Figure 1). This extended along the diaphysis for 2-3 mm proximal and distal to the region of the fracture. Woven bone extended around the entire perimeter of the ulna but was much thicker on the 
medial surface and at the level of the fracture, forming a prominent, focal "callus” centred at the exit point of the fracture. This callus could be detected grossly by visual examination and palpation as well as by histological examination. By 1 week, there was already marked irregular WoB production. Small islands of cartilage were evident within the WoB at the exit point of the fracture at 2 weeks after fracture. At 4 weeks there was marked remodelling of the WoB with bone structure becoming more organised with a less irregular surface. Islands of cartilage were no longer evident within the woven bone. At 6, 8 and 10 weeks the woven bone became progressively more consolidated and organised and there was less remodelling and porosity at each timepoint.

Healing of the fracture line occurred by direct remodelling that originated at the periosteal surface and progressed directly along the fracture plane towards the medullary cavity. The remodelling activity was most exuberant at the exit point of the fracture. Occasionally resorption cavities were also seen emerging from the medullary cavity to extend towards the fracture line at sites distant to the exit point. Basic multicellular units (BMUs) could be followed through serial sections extending along the fracture line towards the centre of the bone (Figure 1). Resorption spaces that appeared to be at a distance from the fracture line in one section could be seen to be associated with the fracture line when followed over serial sections.

Active resorption cavities were first evident at 2 weeks and these originated along the periosteal margin of the fracture plane, especially at the fracture exit, and extending a short way along the fracture line. Numerous osteoclasts lined the crenated leading-edge of the BMUs and osteoblasts formed new lamellar bone along the trailing margin of the BMUs (Figure 1A). At 4 weeks, multiple BMUs had progressed further along the plane of the fracture and new lamellar bone formation had further filled the resorption spaces (Figure 1B). By 6 weeks after loading, the portion of the fracture closest to the exit point was almost 
completely healed (Figure 1C). However, remodelling was not completed, or often not commenced, in the deep region of the fracture, closest to the medullary cavity. Activation of new resorption cavities was dramatically reduced after the 2 week time point and the resorption activity appeared to have slowed or stopped by the 6 week timepoint. At 8 and 10 weeks there was more complete fracture healing (Figure 1D) but there was still minimal evidence of remodelling or healing along the portions of the fracture line closest to the medullary cavity in most bones. No fibrous tissue, cartilage or woven bone was seen along the fracture line at any timepoint. Regions of new bone formation were examined using polarized light and sections stained with Toluidine blue. These confirmed the organisation of new bone along the stress fracture as lamellar bone (data not shown).

\section{TRAP staining}

TRAP staining confirmed the presence of large, multinucleated osteoclasts filling the crenated leading edge of BMUs. Osteoclasts were most abundant at 2 weeks after fracture. A thin line of TRAP staining was seen along the region of the cement line outlining the lamellar bonefilled region that had previously been occupied by a BMU. TRAP staining was also evident along the periosteal margin of the woven bone at 2 and 4 weeks and this was most prominent in the region overlying the fracture (Figure 2).

\section{Histomorphometry}

Histomorphometry results are summarised in (Table 2 and Figure 3). There were no significant differences between groups for cortical bone area (Ct.B.Ar) or total fracture length (SFx.Le) at the transverse section selected for measurement. Woven bone area (Wo.B.Ar) increased from 7 days to 2 weeks and was then reduced again by 4 weeks after loading but these changes were not significant. Wo.B.Ar remained at a similar size for all remaining groups (Table 2). The area and perimeter of BMUs reflected the amount of resorption that had 
occurred to that timepoint. Both these BMU measurements increased rapidly from 2 to 6 weeks after loading with significant differences between these groups. There was minimal additional increase in total size of BMUs after 6 weeks. The area of healed bone within the BMUs (SFx.He.Ar) increased significantly from 2 to 6 weeks but did not have further significant increases between the 6, 8 and 10 week groups (Figure 3). The length of healed fracture continued to increase with each timepoint and was significantly greater at 10 weeks than at 6 weeks. Porosity along the fracture line mainly consisted of resorption space at 2 weeks. This porosity progressively changed to become areas of bone formation by 6 weeks after loading. Porosity area (SFx.Po.Ar) and porosity length (SFx.Po.Le) along the fracture line were greatest at 4 weeks with significant differences between the 4 week group and every other group. An average of $29 \%$ of the fracture had still not undergone resorption or formation at the 10-week timepoint (Figure 1D, Figure 3).

\section{Gene expression}

Fold changes in relative gene expression between loaded and unloaded limbs are summarised in Table 3. In addition, graphs demonstrating quantitative mRNA gene expression relative to GAPDH over 14 days following loading are shown for 10 genes with significant changes in expression in loaded limbs (Figure 4). All genes examined demonstrated significantly increased expression in the right loaded limbs compared to the unloaded control group (ULC) group except for IL-1, Bcl-2, BAX, SOST and RANK. At four hours after fracture there was a marked, 220-fold increase $(\mathrm{P}<0.0001)$ in expression of IL-6 in the right, loaded ulnae compared to the ULC (Figure 4). There were also prominent peak increases in mRNA expression in the right, loaded ulnae compared to the ULC group for OPG, COX-2, and VEGF (all $\mathrm{P}<0.0001$ ) (Figure 4). At 24 hours there was a peak increase in mRNA expression for IL-11 (73-fold increase, $\mathrm{P}<0.0001$ ) (Figure 4). At 4 days there was peak expression of COX-1, OPN, IGF-1, SDF-1, BMP-2, Runx-2 and TNF $\alpha$ which were all significantly 
increased in right, loaded ulnae compared to ULC group (Figure 4). At 7 days there was a significantly increased peak in mRNA expression of RANKL, Collagen-2 in the right, loaded ulnae compared to the ULC group (Figure 4). At 14 days after loading Collagen-10 was significantly increased. Over half of the genes were significantly upregulated in the right ulnae at more than one timepoint. These were IL-6, COX-2, OPG, OPN, IL-11, BMP-2, COX-1, RANKL and Collagen-2.

All unloaded bones and most loaded bones at early timepoints did not express Collagen-2. For those with detectable expression it was very low. All bones in the loaded groups at 7 and 14 days had some expression of Collagen-2 with a significant increase in the average expression in the loaded ulnae at 7 and 14 days compared to the unloaded control group (Table 3). The ratio of expression of RANKL to OPG was significantly decreased in the right loaded ulnae compared to the ULC group at 4 hours. The ratio was then significantly increased at 7 days and 14 days (Figure 4).

In the loaded animals, the expression of COX-1, IGF-1, SOST, SDF-1 and TNF $\alpha$ was also significantly increased in the contralateral (unloaded) left ulna compared to the ULC group at 4 days after fracture. COX-1 and SOST expression was significantly increased in the left ulna at 7 days after stress fracture (Table 4).

\section{Discussion}

This study supports our hypothesis that stress fractures in the rat ulna heal by primary remodelling. It also establishes that remodelling proceeds directly along the fracture line after originating at the periosteal surface. The detailed histological examination clearly demonstrates osteoclasts entering the crack and resorbing bone along the fracture line, followed by osteoblastic lamellar bone formation within the resorption cavity. This pattern of 
stress fracture healing in the rat ulna differs somewhat from classically described direct or primary bone healing, where BMUs emerge from vascular spaces and cross the fracture line [9, 33]. However even in larger mammals, rigidly compressed fractures will contain gaps along the fracture line. These gaps will fill in with new bone originating from the periosteum or endosteum [10, 34] in a similar manner to that seen in this model.

It is interesting that the fatigue fracture is very slow to heal and that there are still areas of fracture present even at 10 weeks after loading. The failure of the deeper regions of the fracture to heal in this model is consistent with prolonged fracture healing seen in many clinical stress fractures and may explain why stress fractures commonly recur [13]. Remodelling activity was much more active at 2 weeks than at the later time points with very few osteoclasts or new resorption cavities evident after the 4 week timepoint. The initiation and vigorous healing of the fracture at its cortical exit point is likely to be due to greater exposure to the periosteum and the associated recruitment of vascular and progenitor cells of fracture healing. Because remodelling proceeds at a given rate, signalling from the fracture line may diminish over time, leading to decreased healing of the fracture line distant to the periosteal origin of BMUs.

Gene expression data correlated well with the histological evidence that most of the active healing occurred in the first 2-4 weeks after fracture. The gene expression results demonstrated prominent upregulation of signalling factors especially within the first 7 days of healing while most increases in gene expression had returned to control levels by 14 days after fracture. Primary fracture healing that occurs with rigid immobility is a much slower process than fracture healing that proceeds via callus formation. The rigid stability created by rapid formation of woven bone may also reduce the stimulus for fracture healing. 
Receptor activator of nuclear factor kappa B ligand(RANKL) is produced by stromal cells and osteoblasts and is the primary ligand responsible for osteoclast differentiation and activation. Osteoprotegerin (OPG), also secreted by osteoblasts and their precursors, acts as a decoy receptor for RANKL and causes inhibition of osteoclastogenesis [35]. The ratio of RANKL/OPG correlates with levels of remodelling and increased RANKL/OPG ratios have been demonstrated in bone sustaining fragility fractures [31, 36]. In the current study, mRNA expression of OPG peaked at 4 hours after loading before slowly declining to normal levels by 14 days. This early increase in expression coincides with a significantly decreased RANKL/OPG ratio at 4 and 24 hours and the early, anabolic period of rapid and dramatic woven bone formation in response to the fatigue fracture. In contrast, RANKL mRNA expression did not increase until 4 days following fracture and peaked at 7 days, just preceding the onset of remodelling along the fracture line. The ratio of RANKL to OPG expression continued to follow the histological appearance of remodelling with the RANKL/OPG ratio significantly increased at 7 and 14 days after fracture when remodelling was becoming most active.

The expression of COX-2 was significantly increased at 4 hours after loading and it remained up-regulated until 4 days after fracture. There was a biphasic increase in expression with distinct peaks occurring at the 4 hour and 4 day timepoints. The Cyclooxygenase (COX) 1 and 2 enzymes are the rate limiting step in the conversion of arachidonic acid to prostanoids [37]. COX-2 is an inducible enzyme and through its production of prostaglandins (PG), especially $\mathrm{PGE}_{2}$, COX-2 plays a central role in both bone formation and bone resorption [37, 38]. Early and sustained upregulation of COX-2 in the current study is consistent with overlapping roles in fracture healing. It is likely that COX-2 is contributing to early bone formation as well as the later remodelling response with its role determined by the changing mechanical, cellular and molecular microenvironment. 
The regulation of COX-1 in bone biology has not been widely examined [37]. Although its level of expression was lower than that of COX-2, COX-1 expression was moderately increased with a significant, 4.7 -fold increase in expression 4 days after fracture. COX-1 is induced by TGFs in osteoblastic MC3T3-E1 cells [39] and the increase of COX-1 in the current study may reflect an abundance of growth factors and high level of cellular activity in response to fracture.

The most dramatic increases in mRNA gene expression were seen with IL-6 and IL-11. IL-6 and IL-11 are cytokines with multiple functions including immune regulation, inflammation, haematopoiesis and oncogenesis. They share the gp130 signal transduction unit along with other members of the IL-6 family of cytokines [40]. IL-6 and IL-11 are co-regulated proresorptive cytokines and have been shown to activate osteoclast activity in RANKL dependant and independent pathways in vitro [40-44]. IL-6 showed a 220-fold increase in expression at only 4 hours after fracture. The dramatic peak expression of a pro-resorptive cytokine in this early, primarily anabolic period of healing is very interesting and points to a broader role of IL-6 in fracture healing and bone remodelling. The magnitude of the IL-6 peak in expression coinciding with the initiation of woven bone formation suggests that IL-6 may be involved in signalling for this new bone. There is in vivo evidence that IL-6 regulates bone formation during fracture healing. For example, localised addition of PTH fragments, IL-6 and the soluble IL-6R to a complete tibial fracture in the rat markedly improved mechanical strength of the fracture compared to control fractures [45].

Expression of IL-11 also increased by 78-fold at 24 hours and 38-fold at 4 days after fracture compared to unloaded controls. IL-11 can also mediate bone formation, when acting in combination with BMP-2 [46]. IL-11 and BMP-2 acted synergistically in vivo to accelerate ectopic bone formation in rats and in repair of segmental bone defects in rabbits [47, 48]. 
Moreover, transgenic mice over-expressing human IL-11 exhibited increased bone formation, without affecting bone resorption or osteoclastogenesis [49]. The role of IL-11 in osteoblastogenesis is possibly mediated by enhancing the effect of BMP-2 and inhibiting bone marrow adipogenesis [49]. Although expression of BMP-2 in the current study peaked at 4 days after loading, expression was already more than doubled by the 4 and 24-hour timepoints consistent with a possible synergistic effect with IL-11 to promote bone formation. It is possible that some of the increase in expression for IL-6, IL-11, COX-2 and VEGF is in response to mechanical strain [38, 50-54]. Ulna loading creates high strains under both tension and compression and the very early expression of these genes could reflect the prolonged cyclic loading that occurs before a fatigue fracture develops. These patterns of gene expression need to be examined in a simple loading model without fatigue to distinguish these functions.

The expression of COX-1, IGF-1, SOST, SDF-1 and TNF $\alpha$ was significantly increased in the unloaded left ulnae compared to the ULC group at 4 days after fracture and SOST and COX-1 expression was significantly increased in the left ulna at 7 days after fracture. These results are intriguing and suggest that systemic factors are exerting effects at sites distant to the fractured limb. An increase in remodelling and bone turnover in the bone adjacent to a fracture was described by Frost [55] as the regional acceleratory phenomenon (RAP) and has been demonstrated in numerous subsequent studies [56-58]. However, a systemic increase in bone turnover is not well recognised. A significant increase in mineral apposition rate and bone formation rate was found in both tibia and a lumbar vertebrae 7 days following the creation of a burr hole defect in only one tibia in a rat model [58]. This provides evidence of a systemic acceleratory phenomenon (SAP) as well as a RAP. More recently, Sample et al (2008) clearly demonstrated periosteal modelling in the ulna and humerus in the opposite, unloaded forelimb following a single bout of ulna loading. This was associated with 
alterations in bone neuropeptide concentrations suggesting neuronal mediation of this systemic response. Increased levels of circulating cytokines, growth factors or hormones are other factors that could be responsible for mediating increased gene expression in bone cells in the opposite limb [59]. Although it is not possible to determine what these factors might be from the current study, increases in gene expression in the limb opposite to the loaded ulna provide further compelling evidence that active fracture healing or remodelling can stimulate systemic signalling for bone turnover that might lead to a SAP.

VEGF is a potent stimulator of angiogenesis and is an important mediator of bone development and regeneration [60]. There was a significant increase in VEGF mRNA expression at 4 hours after loading and expression returned to control levels for the remaining timepoints. In contrast, a peak VEGF expression was seen at 7 days in a rat model of complete fracture healing [23]. Early upregulation of VEGF at 4 hours in the current study is consistent with a previously reported rapid increase in periosteal vascularisation in the first 13 days after fracture $[61,62]$ and an increase in blood flow to the bone at 5 days after loading [17] in the rat ulna model. Wohl et al [63] also demonstrated an increase in expression of VEGF at 24 hours, as well as increases at 4 and 7 days after loading. The increased duration in expression of VEGF compared to the current study may be due to differences in the level of damage induced during loading, and consequent vigour of the woven bone response. As well as stimulation of angiogenesis, necessary for delivery of progenitor cells, VEGF may directly stimulate bone formation by signalling for growth factor production in endothelial cells adjacent to osteoblasts and osteoblast precursors [64]. In addition, VEGF mediated angiogenesis may contribute to early activation of remodelling, as endothelial cell-derived cytokines and growth factors contribute to osteoclast recruitment, delivery and migration into sites of remodelling [60]. This is supported by the significant increase in expression of Stromal cell-derived factor-1 (SDF-1) at 4 days after fracture. SDF-1 is a haemopoietic cell 
chemokine produced by vascular endothelial cells, that has important roles in chemotaxis, early development and survival of osteoclasts [65, 66].

Osteopontin (OPN) is an abundant non-collagenous protein of the extra-cellular matrix of bone and through its interaction with the integrin family of cell adhesion molecules, OPN regulates many cellular actions associated with angiogenesis and mineralisation [67]. OPN is also important in the processes of osteoclast motility and bone resorption [68]. In this study OPN showed 2 peaks in expression with significant increases seen at 4 hours and again at 4 and 7 days after fracture. This pattern of expression highlights the multiple functions of OPN in bone remodelling and tissue mineralisation.

There is considerable evidence that osteocyte apoptosis stimulates "targeted remodelling" of microdamage $[29,69]$ however in the pro-apoptotic factor BAX and the anti-apoptotic factor Bcl-2 did not show increased expression at any timepoint. Positive immunoreactivity has been demonstrated for BAX in osteocytes adjacent to the fracture, and Bcl-2 in cells distant to the fracture seen in this model [29] but the number of osteocytes involved may not be enough to change the gene expression when the whole bone is analysed.

The results from this study display distinct changes in expression of cytokines and growth factors at different stages of fatigue fracture remodelling and demonstrate a clear temporal cascade of signalling events occurring over the first 14 days of healing. The pattern of signalling correlates well with the histological appearance of fracture healing with expression of genes associated with vascularisation, bone formation, osteoclastogenesis and chemotaxis preceding the corresponding cellular activity. The very early period after loading at 4 and 24 hours was characterised by prominent upregulation of IL-6, IL-11, COX-2, OPN and VEGF. These factors are potential mediators of both formation and resorption of bone and are thus 
likely to be involved in initiating a coordinated remodelling and repair response to the fracture.

\section{Acknowledgments}

We thank Mrs Wendy Kelly for expert histotechnical assistance, and Mr Nick Cowling and Ms Nurul Haji Mat Rasil for research assistance. This work was funded in part by NHMRC Project Grants 301143, 401553 and 453624; a University of Queensland Graduate School Scholarship (LK), and The Rebecca L. Cooper Medical Research Foundation. 


\section{References}

\section{Figure Legends}

Figure 1. Transverse sections $(5 \mu \mathrm{m})$ of ulna showing the typical appearance of the stress fracture (arrow head), woven bone (WoB) and remodelling with basic multicellular units (BMU, black arrows) progressing along the fracture from the periosteal margin of the cortex. A. At 2 weeks after loading there is active resorption with a large BMU progressing along the fracture line. There are cuboidal-appearing osteoblasts and a small amount of new bone in the trailing edge of the BMU. B. At 4 weeks remodelling has progressed further along the fracture line. There is still active resorption at the front of the BMU and there is an increased amount of new bone. C. At 6 weeks there is much less active resorption and the BMU is almost completely filled with new bone. D. At 10 weeks there is almost complete healing along the fracture line although there are still small regions of fracture at this level that have not been remodelled (triangular arrow).

Figure 2. (A) TRAP staining 2 weeks after loading. Note the multinucleated osteoclasts (red staining) at the leading edge of the BMUs (arrows) that are progressing along the fracture line (arrow heads). (B) TRAP staining 4 weeks after loading. There are still osteoclasts (red staining) within the BMU (black arrows). New bone formation can now be observed filling the remodelling unit along the fracture line, with TRAP visible at the cement line (arrow heads) (C) TRAP staining 6 weeks after loading. The BMU has largely completed filling of the remodelling unit with TRAP observed at the cement line. Bar $=50 \mathrm{um}$.

Figure 3. Histomorphometry results. A. Graph showing percent (\%) of fracture length occupied by resorption (Resorption length \% = SFx.Po.Le/SFx.Le \%), healed new bone (Healed length \% = SFx.He.Le/SFx.Le \%) and BMU (BMU length \% = BMU.Le/SFx.Le \%).

B. Graph showing area $\left(\mu \mathrm{m}^{2}\right)$ of resorption within the BMUs (Resorption area = SFx.Po.Ar), 
area of healed new bone within the BMUs (Healed area $=$ SFx.He.Ar) and total area of BMUs $(\mathrm{BMU}$ area $=$ BMU.Ar). See table 2 for significant values.

Figure 4. Change in gene expression for (A) IL-6, (B) IL-11, (C) COX-1, (D) COX-2, (E) IGF-1, (F) VEGF, (G) SDF-1, (H) RANKL, (I) OPG; and, (J) ratio of RANKL/OPG in the right loaded (Right) and the opposite left unloaded (Left) ulnae at 4 hours (4h), 24 hours (24h), 4 days (4d), 7 days (7d) and 14 days (14d) after loading. Significant increases in expression for right or left ulnae compared to the separate un-loaded control group (ULC) are designated as ${ }^{\mathrm{a}} \mathrm{P}<0.05 ;{ }^{\mathrm{b}} \mathrm{P} \leq 0.01 ;{ }^{\mathrm{c}} \mathrm{P} \leq 0.001 ;{ }^{\mathrm{d}} \mathrm{P} \leq 0.0001$. mRNA expression is relative to GAPDH. 


\section{References}

[1] Burr DB, Forwood MR, Fyhrie DP, Martin RB, Schaffler MB, Turner CH. Bone microdamage and skeletal fragility in osteoporotic and stress fractures. J Bone Miner Res 11997;12: 6-15.

[2] Muir P, Johnson KA, Ruaux-Mason CP. In vivo matrix microdamage in a naturally occurring canine fatigue fracture. Bone 11999;25: 571-6.

[3] Iwamoto J, Takeda T. Stress fractures in athletes: review of 196 cases. J Orthop Sci 12003;8: 273-8.

[4] Riggs CM. Fractures--a preventable hazard of racing thoroughbreds? Vet J 12002;163: 19-29.

[5] Schaffler MB. Incidence and prevalence of stress fractures in military and athletic populations. In: Burr DB, Milgrom C, editors. Musculoskeletal fatigue and stress fractures; 2001.

[6] Young AJ, McAllister DR. Evaluation and treatment of tibial stress fractures. Clin Sports Med 12006;25: 117-28, x.

[7] Tsangari H, Findlay DM, Zannettino AC, Pan B, Kuliwaba JS, Fazzalari NL. Evidence for reduced bone formation surface relative to bone resorption surface in female femoral fragility fracture patients. Bone 12006;39: 1226-35.

[8] Mori S, Li J, Kawaguchi H. The histological appearance of stress fractures. In: Burr DB, Milgrom C, editors. Musculoskeletal fatigue and stress fractures: CRC Press; 2001, p. 151-160.

[9] Einhorn TA. The cell and molecular biology of fracture healing. Clin Orthop Relat Res 11998: S7-21.

[10] McKibbin B. The biology of fracture healing in long bones. J Bone Joint Surg $\mathrm{Br}$ 11978;60-B: 150-62.

[11] Stover SM, Johnson BJ, Daft BM, Read DH, Anderson M, Barr BC, Kinde H, Moore J, Stoltz J, Ardans AA, et al. An association between complete and incomplete stress fractures of the humerus in racehorses. Equine Vet J 11992;24: 260-3.

[12] Hasselman CT, Gruen GS. Principles of operative fracture stabilization and fixation. In: Fitzgerald RH, Kaufer H, Malkani AL, editors. Orthopaedics: Mosby; 2002, p. 228-238.

[13] Peris P. Stress fractures. Best Pract Res Clin Rheumatol 12003;17: 1043-61.

[14] Rolf C, Ekenman I, Tornqvist H, Gad A. The anterior stress fracture of the tibia: an atrophic pseudoarthosis? Scand J Med Sci Sports 11997; 7: 249-52. 
[15] Bentolila V, Boyce TM, Fyhrie DP, Drumb R, Skerry TM, Schaffler MB. Intracortical remodeling in adult rat long bones after fatigue loading. Bone 11998;23: 275-81.

[16] Danova NA, Colopy SA, Radtke CL, Kalscheur VL, Markel MD, Vanderby R, McCabe RP, Escarcega AJ, Muir P. Degradation of bone structural properties by accumulation and coalescence of microcracks. Bone 12003;33: 197-205.

[17] Muir P, Sample SJ, Barrett JG, McCarthy J, Vanderby R, Jr., Markel MD, Prokuski LJ, Kalscheur VL. Effect of fatigue loading and associated matrix microdamage on bone blood flow and interstitial fluid flow. Bone 12007;40: 948-56.

[18] Uthgenannt BA, Silva MJ. Use of the rat forelimb compression model to create discrete levels of bone damage in vivo. J Biomech 12007;40: 317-24.

[19] Hsieh YF, Silva MJ. In vivo fatigue loading of the rat ulna induces both bone formation and resorption and leads to time-related changes in bone mechanical properties and density. J Orthop Res 12002;20: 764-71.

[20] Tami AE, Nasser P, Schaffler MB, Knothe Tate ML. Noninvasive fatigue fracture model of the rat ulna. J Orthop Res 12003;21: 1018-24.

[21] Li J, Waugh LJ, Hui SL, Burr DB, Warden SJ. Low-intensity pulsed ultrasound and nonsteroidal anti-inflammatory drugs have opposing effects during stress fracture repair. $\mathrm{J}$ Orthop Res 12007;25: 1559-67.

[22] Kuroda S, Virdi AS, Dai Y, Shott S, Sumner DR. Patterns and localization of gene expression during intramembranous bone regeneration in the rat femoral marrow ablation model. Calcif Tissue Int 12005;77: 212-25.

[23] Meyer RA, Meyer MH, Phieffer LS, Banks DM. Delayed union of femoral fractures in older rats:decreased gene expression. BMC Musculoskelet Disord 12001;2: 2.

[24] Einhorn TA, Majeska RJ, Rush EB, Levine PM, Horowitz MC. The expression of cytokine activity by fracture callus. J Bone Miner Res 11995;10: 1272-81.

[25] Mori S, Burr DB. Increased intracortical remodeling following fatigue damage. Bone 11993;14: 103-9.

[26] Colopy SA, Benz-Dean J, Barrett JG, Sample SJ, Lu Y, Danova NA, Kalscheur VL, Vanderby R, Jr., Markel MD, Muir P. Response of the osteocyte syncytium adjacent to and distant from linear microcracks during adaptation to cyclic fatigue loading. Bone 12004;35: 881-91.

[27] Noble BS, Peet N, Stevens HY, Brabbs A, Mosley JR, Reilly GC, Reeve J, Skerry TM, Lanyon LE. Mechanical loading: biphasic osteocyte survival and targeting of osteoclasts for bone destruction in rat cortical bone. Am J Physiol Cell Physiol 12003;284: C934-43. 
[28] Noble BS, Stevens H, Loveridge N, Reeve J. Identification of apoptotic changes in osteocytes in normal and pathological human bone. Bone 11997;20: 273-82.

[29] Verborgt O, Tatton NA, Majeska RJ, Schaffler MB. Spatial distribution of Bax and $\mathrm{Bcl}-2$ in osteocytes after bone fatigue: complementary roles in bone remodeling regulation? $\mathrm{J}$ Bone Miner Res 12002;17: 907-14.

[30] Verborgt O, Gibson GJ, Schaffler MB. Loss of osteocyte integrity in association with microdamage and bone remodeling after fatigue in vivo. J Bone Miner Res 12000;15: 60-7.

[31] Fazzalari NL, Kuliwaba JS, Atkins GJ, Forwood MR, Findlay DM. The ratio of messenger RNA levels of receptor activator of nuclear factor kappaB ligand to osteoprotegerin correlates with bone remodeling indices in normal human cancellous bone but not in osteoarthritis. J Bone Miner Res 12001;16: 1015-27.

[32] Forwood MR, Bennett MB, Blowers AR, Nadorfi RL. Modification of the in vivo four-point loading model for studying mechanically induced bone adaptation. Bone 11998;23: 307-10.

[33] Perren SM, Huggler A, Russenberger M, Allgower M, Mathys R, Schenk R, Willenegger H, Muller ME. The reaction of cortical bone to compression. Acta Orthop Scand Suppl 11969;125: 19-29.

[34] Olerud S, Danckwardt-Lilliestrom G. Fracture healing in compression osteosynthesis in the dog. J Bone Joint Surg Br 11968;50: 844-51.

[35] Hofbauer LC, Heufelder AE. Role of receptor activator of nuclear factor-kappaB ligand and osteoprotegerin in bone cell biology. J Mol Med 12001;79: 243-53.

[36] Abdallah BM, Stilgren LS, Nissen N, Kassem M, Jorgensen HR, Abrahamsen B. Increased RANKL/OPG mRNA ratio in iliac bone biopsies from women with hip fractures. Calcif Tissue Int 12005;76: 90-7.

[37] Li L, Pettit AR, Gregory LS, Forwood MR. Regulation of bone biology by prostaglandin endoperoxide H synthases (PGHS): a rose by any other name. Cytokine Growth Factor Rev 12006;17: 203-16.

[38] Forwood MR. Inducible cyclo-oxygenase (COX-2) mediates the induction of bone formation by mechanical loading in vivo. J Bone Miner Res 11996;11: 1688-93.

[39] Pilbeam CC, Kawaguchi H, Hakeda Y, Voznesensky O, Alander CB, Raisz LG. Differential regulation of inducible and constitutive prostaglandin endoperoxide synthase in osteoblastic MC3T3-E1 cells. J Biol Chem 11993;268: 25643-9.

[40] Heymann D, Rousselle AV. gp130 Cytokine family and bone cells. Cytokine 12000;12: 1455-68. 
[41] Kudo O, Sabokbar A, Pocock A, Itonaga I, Fujikawa Y, Athanasou NA. Interleukin-6 and interleukin-11 support human osteoclast formation by a RANKL-independent mechanism. Bone 12003;32: 1-7.

[42] Sims NA, Jenkins BJ, Nakamura A, Quinn JM, Li R, Gillespie MT, Ernst M, Robb L, Martin TJ. Interleukin-11 receptor signaling is required for normal bone remodeling. J Bone Miner Res 12005;20: 1093-102.

[43] Palmqvist P, Persson E, Conaway HH, Lerner UH. IL-6, leukemia inhibitory factor, and oncostatin $\mathrm{M}$ stimulate bone resorption and regulate the expression of receptor activator of NF-kappa B ligand, osteoprotegerin, and receptor activator of NF-kappa B in mouse calvariae. J Immunol 12002;169: 3353-62.

[44] Tsangari H, Findlay DM, Kuliwaba JS, Atkins GJ, Fazzalari NL. Increased expression of IL-6 and RANK mRNA in human trabecular bone from fragility fracture of the femoral neck. Bone 12004;35: 334-42.

[45] Rozen N, Lewinson D, Bick T, Jacob ZC, Stein H, Soudry M. Fracture repair: modulation of fracture-callus and mechanical properties by sequential application of IL-6 following PTH 1-34 or PTH 28-48. Bone 12007;41: 437-45.

[46] Suga K, Saitoh M, Fukushima S, Takahashi K, Nara H, Yasuda S, Miyata K. Interleukin-11 induces osteoblast differentiation and acts synergistically with bone morphogenetic protein-2 in C3H10T1/2 cells. J Interferon Cytokine Res 12001;21: 695-707.

[47] Suga K, Saitoh M, Kokubo S, Fukushima S, Kaku S, Yasuda S, Miyata K. Interleukin11 acts synergistically with bone morphogenetic protein-2 to accelerate bone formation in a rat ectopic model. J Interferon Cytokine Res 12003;23: 203-7.

[48] Suga K, Saitoh M, Kokubo S, Nozaki K, Fukushima S, Yasuda S, Sasamata M, Miyata K. Synergism between interleukin-11 and bone morphogenetic protein-2 in the healing of segmental bone defects in a rabbit model. J Interferon Cytokine Res 12004;24: 3439.

[49] Takeuchi Y, Watanabe S, Ishii G, Takeda S, Nakayama K, Fukumoto S, Kaneta Y, Inoue D, Matsumoto T, Harigaya K, Fujita T. Interleukin-11 as a stimulatory factor for bone formation prevents bone loss with advancing age in mice. J Biol Chem 12002;277: 49011-8.

[50] Thi MM, Iacobas DA, Iacobas S, Spray DC. Fluid shear stress upregulates vascular endothelial growth factor gene expression in osteoblasts. Ann N Y Acad Sci 12007;1117: 7381. 
[51] Cillo JE, Jr., Gassner R, Koepsel RR, Buckley MJ. Growth factor and cytokine gene expression in mechanically strained human osteoblast-like cells: implications for distraction osteogenesis. Oral Surg Oral Med Oral Pathol Oral Radiol Endod 12000;90: 147-54.

[52] Koyama Y, Mitsui N, Suzuki N, Yanagisawa M, Sanuki R, Isokawa K, Shimizu N, Maeno M. Effect of compressive force on the expression of inflammatory cytokines and their receptors in osteoblastic Saos-2 cells. Arch Oral Biol 12008.

[53] Wadhwa S, Choudhary S, Voznesensky M, Epstein M, Raisz L, Pilbeam C. Fluid flow induces COX-2 expression in MC3T3-E1 osteoblasts via a PKA signaling pathway. Biochem Biophys Res Commun 12002;297: 46-51.

[54] Singh SP, Chang EI, Gossain AK, Mehara BJ, Galiano RD, Jensen J, Longaker MT, Gurtner GC, Saadeh PB. Cyclic mechanical strain increases production of regulators of bone healing in cultured murine osteoblasts. J Am Coll Surg 12007;204: 426-34.

[55] Frost HM. The regional acceleratory phenomenon: a review. Henry Ford Hosp Med J 11983;31: 3-9.

[56] Rohde C, Anderson DE, Bertone AL, Weisbrode SE. Effects of phenylbutazone on bone activity and formation in horses. Am J Vet Res 12000;61: 537-43.

[57] Schilling T, Muller M, Minne HW, Ziegler R. Influence of inflammation-mediated osteopenia on the regional acceleratory phenomenon and the systemic acceleratory phenomenon during healing of a bone defect in the rat. Calcif Tissue Int 11998;63: 160-6.

[58] Mueller M, Schilling T, Minne HW, Ziegler R. A systemic acceleratory phenomenon (SAP) accompanies the regional acceleratory phenomenon (RAP) during healing of a bone defect in the rat. J Bone Miner Res 11991;6: 401-10.

[59] Sample SJ, Behan M, Smith L, Oldenhoff WE, Markel M, Kalscheur VL, Hao Z, Miletic V, Muir P. Functional Adaptation to Loading of a Single Bone is Neuronally Regulated and Involves Multiple Bones. J Bone Miner Res 12008.

[60] Brandi ML, Collin-Osdoby P. Vascular biology and the skeleton. J Bone Miner Res 12006;21: 183-92.

[61] Silva MJ, Uthgenannt BA, Rutlin JR, Wohl GR, Lewis JS, Welch MJ. In vivo skeletal imaging of 18F-fluoride with positron emission tomography reveals damage- and timedependent responses to fatigue loading in the rat ulna. Bone 12006;39: 229-36.

[62] Matsuzaki H, Wohl GR, Novack DV, Lynch JA, Silva MJ. Damaging fatigue loading stimulates increases in periosteal vascularity at sites of bone formation in the rat ulna. Calcif Tissue Int 12007;80: 391-9. 
[63] Wohl GR, Towler DA, Silva MJ. Stress fracture healing: fatigue loading of the rat ulna induces upregulation in expression of osteogenic and angiogenic genes that mimic the intramembranous portion of fracture repair. Bone 12009;44: 320-30.

[64] Wang DS, Miura M, Demura H, Sato K. Anabolic effects of 1,25-dihydroxyvitamin D3 on osteoblasts are enhanced by vascular endothelial growth factor produced by osteoblasts and by growth factors produced by endothelial cells. Endocrinology 11997;138: 2953-62.

[65] Gronthos S, Zannettino AC. The role of the chemokine CXCL12 in osteoclastogenesis. Trends Endocrinol Metab 12007;18: 108-13.

[66] Wright LM, Maloney W, Yu X, Kindle L, Collin-Osdoby P, Osdoby P. Stromal cellderived factor-1 binding to its chemokine receptor CXCR4 on precursor cells promotes the chemotactic recruitment, development and survival of human osteoclasts. Bone 12005;36: 840-53.

[67] Duvall CL, Taylor WR, Weiss D, Wojtowicz AM, Guldberg RE. Impaired angiogenesis, early callus formation, and late stage remodeling in fracture healing of osteopontin-deficient mice. J Bone Miner Res 12007;22: 286-97.

[68] Chellaiah MA, Kizer N, Biswas R, Alvarez U, Strauss-Schoenberger J, Rifas L, Rittling SR, Denhardt DT, Hruska KA. Osteopontin deficiency produces osteoclast dysfunction due to reduced CD44 surface expression. Mol Biol Cell 12003;14: 173-89.

[69] Noble B. Microdamage and apoptosis. Eur J Morphol 12005;42: 91-8. 